\title{
P I 5-08. Did unblinding affect HIV risk behaviour and risk perception in the HVTN503/Phambili study?
}

\author{
GE Gray*1, L Bekker ${ }^{2}$, G Churchyard ${ }^{3}$, M Nchabeleng 4 , K Mlisana ${ }^{5}$, G de \\ Bruyn ${ }^{1}$, S Roux ${ }^{2}$, M Mathebula ${ }^{4}, \mathrm{M} \mathrm{Latka}^{3}$, T Bennie ${ }^{4}$, B Metch ${ }^{6}, \mathrm{Z}^{5}$ Moodie ${ }^{6}$, \\ M Allen ${ }^{7}, \mathrm{~N}$ Eaton ${ }^{8}$ and J Kublin ${ }^{8}$
}

Address: ${ }^{1}$ Perinatal HIV Research Unit, Soweto, South Africa, ${ }^{2}$ Desmond Tutu HIV Foundation, Cape Town, South Africa, ${ }^{3}$ Aurum Institute for Health Research, Cape Town, South Africa, ${ }^{4}$ Medunsa HIV Clinical Research Unit (MeCRU), Tswane, South Africa, ${ }^{5}$ Centre for the AIDS Programme for Research in South Africa (CAPRISA), Durban, South Africa, ${ }^{6}$ Statistical Center for HIV/AIDS Research and Prevention, FHCRC, Seattle, WA, USA, ${ }^{7}$ National Institute of Allergy and Infectious Diseases, NIH, Bethesda, MD USA and ${ }^{8}$ HIV Vaccine Trials Network, Fred Hutchinson Cancer Research Center, Seattle, WA, USA

* Corresponding author

from AIDS Vaccine 2009

Paris, France. 19-22 October 2009

Published: 22 October 2009

Retrovirology 2009, 6(Suppl 3):P209 doi:10.1 186/I742-4690-6-S3-P209

This abstract is available from: http://www.retrovirology.com/content/6/S3/P209

(c) 2009 Gray et al; licensee BioMed Central Ltd.

\section{Background}

The HVTN 503/Phambili study, a phase IIB study of the Merck Ad-5 multiclade HIV vaccine, suspended enrollment and vaccinations following the results of the HVTN502/STEP study. Participants were notified of their treatment allocation and continue to be followed. We investigate the impact of study unblinding on HIV risk perception and behaviour.

\section{Methods}

Before suspension, 801 participants were enrolled. Data from all participants who had a post-treatment notification behaviour risk assessment (PTN-RA) were compared with the baseline RA. In addition an unblinding risk perception questionnaire was administered post-treatment notification (PTN).

\section{Results}

469 participants were eligible for risk behaviour analysis: median time between unblinding and administration of PTN-RA being 70 days (IQR: 39-108 days). At baseline, amongst men, there were no statistical differences in risk behaviour reported by treatment arm, and borderline differences in unprotected anal sex with a female partner ( $0 \%$ vaccinee vs. $2.9 \%$ placebo arm, $\mathrm{p}=0.056)$, and a STI diagnosis $(7.7 \%$ vaccinee vs. $2.2 \%$ placebo, $\mathrm{p}=0.052)$. For women, there were no differences in risk behaviour by treatment arm at baseline. Post-treatment notification, risk behaviour change was similar for both sexes, with the pattern of change being similar in both arms. Between screening and the PTN-RA, significant reductions in number of sexual partners $(\mathrm{p}<0.001)$, and unprotected sex $(p<0.001)$. were observed. 677 participants completed the unblinding risk perception questionnaire. In this assessment, men were more likely to report behaviour change $(22.0 \%)$ than women $(14.1 \%)$. Men reported increased condom use $(64.6 \%)$, encouraging partner testing (51.2\%) and reduced partner numbers (57.3\%). More vaccinees $(24.7 \%)$ as compared to placebo recipients $(12.1 \%)$ agreed/strongly agreed that they were more likely to get HIV than most people $(\mathrm{p}<0.001)$, which was attributed by the participant to receiving the vaccine.

\section{Conclusion}

Post-unblinding, vaccinees perceived being at increased risk of HIV, but risk behaviour reductions were noted irrespective of treatment allocation. 